\title{
A MAJORANT PROBLEM
}

\author{
RONEN PERETZ
}

Department of Mathematics

University of Michigan

Ann Arbor, MI $\mathbf{4 8 1 0 9}$

(Received September 25, 1990)

ABSTRACT. Let $f(z)=\sum_{k=0}^{\infty} a_{k} z^{k}, a_{0} \neq 0$ be analytic in the unit disc. Any infinite complex vector $\theta=\left(\theta_{0}, \theta_{1}, \theta_{2}, \ldots\right)$ such that $\left|\theta_{k}\right|=1$, $k=0,1,2, \ldots$, induces a function $f_{\theta}(z)=\sum_{k=0}^{\infty} a_{k} \theta_{k} z^{k}$ which is still analytic in the unit disc.

In this paper we study the problem of maximizing the p-means:

$$
\int_{0}^{2 \pi}\left|f_{\theta}\left(\mathrm{re}^{i \phi}\right)\right|^{p} \mathrm{d \phi}
$$

over all possible vectors $\theta$ and for values of $r$ close to 0 and for all $p<2$.

It is proved that a maximizing function is $f_{1}(z)=-\left|a_{0}\right|+\sum_{k=1}^{\infty}\left|a_{k}\right| z^{k}$ and that $r$ could be taken to be any positive number which is smaller than the radius of the largest disc centered at the origin which can be inscribed in the zero sets of $f_{1}$. This problem is originated by a well known majorant problem for Fourier coefficients that was studied by Hardy and Littlewood.

One consequence of our paper is that for $p<2$ the extremal function for the Hardy-Littlewood problem should be $-\left|a_{0}\right|+\sum_{k=1}^{\infty}\left|a_{k}\right| z^{k}$.

We also give some applications to derive some sharp inequalities for the classes of Schlicht functions and of functions of positive real part.

Key Words and Phrases: Fourier coefficients, analytic continuation, Schlicht functions, functions of positive real part

1980 AMS SUBJECT CLASSIFICATION CODES: 42A05, 30B10, $30 \mathrm{D5} 5$

1. DEFINITIONS AND NOTATIONS.

We will denote $D(a, r)=\{z \in C|| z-a \mid<r\}$ and $U=D(0,1)$.

$H(U)$ is the set of all functions analytic in $U$.

If $f(z)=\sum_{k=0}^{\infty} a_{k} z^{k} \in H(U)$ and if $\theta=\left(\theta_{0}, \theta_{1}, \theta_{2}, \ldots\right)$ is an infinite complex vector such that $\left|\theta_{k}\right|=1, k=0,1,2, \ldots$, then we will define 


$$
f_{\theta}(z)=\sum_{k=0}^{\infty}\left|a_{k}\right| \theta_{k} z^{k}, z \in U .
$$

For fixed $p$ and $r$ such that $p \in R$ and $0 \leq r<1$, we will define $a$ functional on $\left\{f_{\theta}\right\}$ by the formula: $J_{p, r}(\theta)=\int_{0}^{2 \pi}\left|f_{\theta}\left(r e^{i \phi}\right)\right|^{p} d \phi$.

If $n \in Z$ then we will denote: $c_{f, p, n}(r)=\frac{1}{2 \pi} \int_{0}^{2 \pi}\left|f\left(r e^{i \phi}\right)\right|^{p} e^{-i n \phi} d \phi$. (Usually $f$ and $p$ will be fixed so we will shorten $c_{n}(r)=c_{f, p, n}(r)$ ).

The radius of the zero-free region of $f$ will be defined by

$$
\delta(f)=\min (1, \min (\{|z| \mid z \neq 0, f(z)=0\})) .
$$

If $F(z)=\sum_{k=0}^{\infty} A_{k} z^{k} \in H(U)$ and satisfies $\left|a_{k}\right| \leq A_{k}, \quad k=0,1,2, \ldots$. then we will say that $F$ majorizes $f$ and write $f$ \& $F$.

Some familiar families of analytic functions in $U$ are:

$S=\left\{f \in H(U) \mid f(0)=0, f^{\prime}(0)=1, \quad f\right.$ is univalent in $\left.U\right\}$

$P=\{f \in H(U) \mid f(0)=1, \quad \operatorname{Re}\{f(z)\}>0 \forall z \in U\}$.

2. THE MAIN RESULTS.

$$
\begin{aligned}
\text { If } f(z) & =\sum_{k=m}^{\infty} a_{k} z^{k} \in H(U), a_{m} \neq 0 . \text { Denote } f_{1}(z)=-\left|a_{m}\right| z^{m}+\sum_{k=1}^{\infty}\left|a_{m+k}\right|^{m+k} . \\
\text { Let } f_{\alpha}(z) & =-1+\sum_{k=1}^{\infty} \alpha_{k} z^{k}, f_{\beta}(z)=-1+\sum_{k=1}^{\infty} \beta_{k} z^{k} \in H(U) \text { satisfy } \\
0 \leq \alpha_{k} \leq \beta_{k}, \quad k & =1,2,3, \ldots
\end{aligned}
$$

The following theorems will be proved in the paper.

THEOREM A. For every $p<2$ and every $0 \leq r<\delta\left(f_{1}\right)$, we have

$$
\int_{0}^{2 \pi}\left|f\left(r e^{i \phi}\right)\right|^{p} d \phi \leq \int_{0}^{2 \pi}\left|f_{1}\left(r e^{i \phi}\right)\right|^{p} d \phi .
$$

The inequality is usually not valid for $r>\delta\left(f_{1}\right)$.

THEOREM B. For every $p<2$ and every $0 \leq r<\delta\left(f_{\beta}\right)$, we have

$$
\int_{0}^{2 \pi}\left|f_{\alpha}\left(r e^{1 \phi}\right)\right|^{p} d \phi \leq \int_{0}^{2 \pi}\left|f_{\beta}\left(r e^{1 \phi}\right)\right|^{p} d \phi .
$$

THEOREM C. For every $p<0$ and $0 \leq r<\delta\left(f_{1}\right)$, and for all $\mathrm{n}=1,2,3, \ldots$, we have $\left.\left.\left|\int_{0}^{2 \pi}\right| \mathrm{f}\left(\mathrm{re} \mathrm{e}^{i \phi}\right)\right|^{\mathrm{p}} \mathrm{e}^{-i n \phi} \mathrm{d \phi}\left|\leq \int_{0}^{2 \pi}\right| \mathrm{f}_{1}\left(r \mathrm{e}^{i \phi}\right)\right|^{\mathrm{p}} \mathrm{e}^{-i n \phi} \mathrm{d \phi}$.

THEOREM D. For every $p<0$ and every $0 \leq r<\delta\left(f_{\beta}\right)$, and for all $\mathrm{n}=1,2,3, \ldots$, we have

$$
\int_{0}^{2 \pi}\left|f_{\alpha}\left(r e^{i \phi}\right)\right|^{p} e^{-i n \phi} d \phi \leq \int_{0}^{2 \pi}\left|f_{\beta}\left(r e^{i \phi}\right)\right|^{p} e^{-i n \phi} d \phi .
$$

\section{A MAJORANT PROBLEM DUE TO HARDY AND LITTLEWOOD.}

In this paper we solve the following extremal problem: $\max J_{p, r}(\theta)$, for every $p<2$ and every $0 \leq r<\delta\left(f_{1}\right)$. We also find the extremal directions $\theta^{\prime}$ for which $J_{p, r}\left(\theta^{\prime}\right)=\underset{\theta}{\max } J_{p, r}(\theta)$. (The compactness of $\left\{f_{\theta}\right\}$ in the topology of uniform convergence on compact 
subsets of $U$ guarantees the existence of maximizing functions). A majorant problem for Fourier coefficients, that was studied by Hardy and Littlewood, goes as follows:

If $p$ is an even integer and if $f \ll F$ and $F \in L^{p}$, then $f \in L^{p}$ and, in fact, $\|f\|_{p} \leq\|F\|_{p}$.

Are there any other values of $p$ for which an inequality of the type $\|f\|_{p} \leq B_{p}\|F\|_{p}$ holds whenever $f \ll F$ ?

In his paper [2] from 1975, Harold Shapiro characterizes the class of all smooth functions $\Phi$ on $\mathbf{R}^{+}$for which $\int_{0}^{2 \pi} \Phi\left(\left|f\left(e^{i t}\right)\right|\right) d t$ $\leq \int_{0}^{2 \pi} \Phi\left(\left|F\left(e^{i t}\right)\right|\right) d t$. In particular he deduces that $\Phi(x)=x^{p}$ is not such a function unless $p$ is an even integer.

One consequence of our paper in connection with this problem is that for $p<2$ the majorant function of Hardy and Littlewood should be replaced by a new majorant function:

$$
-\left|a_{0}\right|+\sum_{k=1}^{\infty}\left|a_{k}\right| z^{k} \text { instead of } \sum_{k=0}^{\infty}\left|a_{k}\right| z^{k} \quad\left(a_{0} \neq 0\right) \text {. }
$$

\section{PRELIMINARY REMARKS.}

(a) If $f, g \in H(u)$ and $f * g$, then for every $p=2 k, k=0,1,2, \ldots$ and every $0 \leq r<1$ we have

$$
\int_{0}^{2 \pi}\left|f\left(r e^{i \phi}\right)\right|^{p} d \phi \leq \int_{0}^{2 \pi}\left|g\left(r e^{i \phi}\right)\right|^{p} d \phi
$$

Even more is true:

$$
\left.\left.\left|\int_{0}^{2 \pi}\right| f\left(r e^{i \phi}\right)\right|^{p} e^{-i n \phi} d \phi\left|\leq \int_{0}^{2 \pi}\right| g\left(r e^{i \phi}\right)\right|^{p} e^{-i n \phi} d \phi, \quad n=0,1,2, \ldots .
$$

(In view of the fact that for every $f \in S$ and every $h \in P$ we have

we can deduce that

$$
f * k(z)=\frac{z}{(1-z)^{2}}, h \ll \ell(z)=\frac{1+z}{1-z}
$$

$$
\begin{array}{ll}
\int_{0}^{2 \pi}\left|f^{(n)}\left(r e^{i \phi}\right)\right|^{2 k} d \phi \leq \int_{0}^{2 \pi}\left|k^{(n)}\left(r e^{i \phi}\right)\right|^{2 k} d \phi, & f \in S \\
\int_{0}^{2 \pi}\left|h^{(n)}\left(r e^{i \phi}\right)\right|^{2 k} d \phi \leq \int_{0}^{2 \pi}\left|\ell^{(n)}\left(r e^{i \phi}\right)\right|^{2 k} d \phi, & h \in P
\end{array}
$$

for all $n, k=0,1,2, \ldots$ and $0 \leq r<1$ )

(b) If $f(z)=\sum_{k=0}^{\infty} a_{k} z^{k} \in H(U), a_{0} \neq 0$ and if $f_{1}(z)=-\left|a_{0}\right|+\sum_{k=1}^{\infty}\left|a_{k}\right| z^{k}$ then (by the triangle inequality) we have

$$
\min _{0 \leq \phi \leq 2 \pi}\left|f_{1}\left(r e^{i \phi}\right)\right| \leq \min _{0 \leq \phi \leq 2 \pi}\left|f\left(r e^{i \phi}\right)\right|, \quad 0 \leq r<\delta\left(f_{1}\right)
$$

and in particular $\delta\left(f_{1}\right) \leq \delta(f)$.

(c) If $p \neq 0,0 \leq r<\delta\left(f_{1}\right)$ and $\theta^{\prime}=\left(\theta_{0}, \theta_{1}, \theta_{2}, \ldots\right)$ satisfies $J_{p, r}\left(\theta^{\prime}\right)=\max _{\theta} J_{p, r}(\theta)$, and if we have the Fourier expansions: 


$$
\begin{gathered}
f_{\theta^{\prime}}(z)=\sum_{k=0}^{\infty}\left|a_{k}\right| \theta_{k} z^{k} \\
\left|f_{\theta^{\prime}}(z)\right|^{p-2} f_{\theta^{\prime}}(z) \sim \sum_{k=-\infty}^{\infty} b_{k} z^{k} \\
\end{gathered}
$$

then for all $k=0,1,2, \ldots$, we have $\arg \left(b_{k}\right) \in\left\{\arg \left(\theta_{k}\right), \arg \left(\theta_{k}\right)+\pi\right\}$. (To see this we use the fact that for all $k=0,1,2, \ldots$ we have $\frac{\partial J_{p, r}\left(\theta^{\prime}\right)}{\partial \theta_{k}}=0$.)

5. AN ANALYTIC EXTENSION FOR $c_{n}(r)$.

PROPOSITION 1. Let $f \in H(U)$ and $n \in Z$.

(a) If $p \in\{2 k \mid k=0,1,2, \ldots\}$ then there is a $g_{n} \in H(U)$ such that $g_{n}(r)=$ $c_{f, p, n}(r)$ for every $0 \leq r<1$.

(b) If $p \in\{2 k \mid k=0,1,2, \ldots\}$ and if $f$ has a zero of order $m$ at $z=0$, then there is a $g_{n} \in H(D(0, \delta(f)))$ such that $r^{m p} g_{n}(r)=c_{f, p, n}(r)$ for every $0 \leq r<\delta(f)$. Moreover for each $\eta>\delta(f)$ we have $g_{n} \notin H(D(0, \eta))$.

Proof. It is enough to consider the case $n=0$.

(a) If $f(z)=\sum_{l=0}^{\infty} a_{l} z^{l}$ then $f(z)^{k}=\sum_{l=0}^{\infty} p_{l}\left(a_{0}, \ldots, a_{l}\right) z^{l}$ where $p_{l}$ is an homogeneous polynomial of degree $k$ with positive integer coefficients such that $\overline{11 \mathrm{~m}}\left|\mathrm{P}_{\ell}\right|^{1 / \ell}=\overline{1 \mathrm{~m}}\left|\mathrm{a}_{\ell}\right|^{1 / \ell}$. By Parseval's equality the desired analytic extension of $c_{0}(r)$ is given by $g_{0}(z)=\sum_{\ell=0}^{\infty}\left|p_{\ell}\left(a_{0}, \ldots, a_{\ell}\right)\right|^{2} z^{2 \ell}$.

(b) Let $h \in H(U)$ satisfy $h(0) \neq 0$ and $f(z)=z^{m} h(z), \quad z \in U$. Since $\delta(h)=\delta(f)$ we have for each fixed $\phi \in R$ that $\left(h\left(z e^{1 \phi}\right) \overline{\left.h\left(\bar{z} e^{1 \phi}\right)\right)^{p / 2}}\right.$ is an analytic function in $\mathrm{D}(0, \delta(f))$. Hence $g_{0}(z)=\frac{1}{2 \pi} \int_{0}^{2 \pi}\left(\mathrm{h}\left(\mathrm{ze}^{1 \phi}\right) \overline{\mathrm{h}\left(\overline{\mathrm{z}} \mathrm{e}^{1 \phi}\right)}\right)^{\mathrm{p} / 2} \mathrm{d \phi}$ is an analytic function in $D(0, \delta(f))$ and clearly $r^{m p} g_{0}(r)=c_{0}(r)$ for $0 \leq r<\delta(f)$. Finally, if $\left(h\left(z e^{1 \phi}\right)\right)^{p / 2}=\sum_{l=0}^{\infty} d_{l}\left(z e^{1 \phi}\right)^{l},|z|<\delta(f)$ then by Parseval's equality we have

But $\left.\left.\overline{\operatorname{Im}}|| \mathrm{d}_{\ell}\right|^{2}\right|^{\frac{1}{2} \ell}=\overline{1 \mathrm{im}}\left|\mathrm{d}_{\ell}\right|^{1 / \ell}=\frac{1}{\delta(\mathrm{f})}$ because $\left(\mathrm{h}\left(\mathrm{ze} \mathrm{e}^{1 \phi}\right)\right)^{\mathrm{p} / 2}$ has a singular point on $|z|=\delta(f)$ (since $\frac{p}{2}$ is not a nonnegative integer).

We remark that in (b) we have proved the representation

$$
\frac{1}{2 \pi} \int_{0}^{2 \pi}\left|f\left(r e^{1 \phi}\right)\right|^{p_{d}}=r^{m p} \sum_{l=0}^{\infty}\left|d_{l}\right|^{2} z^{2 \ell}, \quad 0 \leq r<\delta(f) .
$$

6. A SOLUTION FOR THE PROBLEM $\max _{\theta} J_{p, r}(\theta)$ FOR $p<2,0 \leq r<\delta(f)$.

Let $f(z)=1+\sum_{k=1}^{\infty} \alpha_{k} z^{k} \in H(U)$ satisfy $0<f$. Let $\theta=\left(1, \theta_{1}, \theta_{2}, \ldots\right)$ 
be an infinite complex vector such that $\left|\theta_{k}\right|=1, k=1,2,3, \ldots$. Suppose $q$ $<1$ and $\left(f_{\theta}(z)\right)^{q}=\sum_{k=0}^{\infty} d_{\theta k} z^{k},|z|<\delta\left(f_{\theta}\right)$.

LEMA 2. If $\psi=(1,1,-1,1,-1,1,-1, \ldots)$ then for every $\theta$ we have

$$
\left|d_{\theta k}\right| \leq\left|d_{\psi k}\right|, \quad k=1,2,3, \ldots
$$

Proof. Denote $x=\sum_{k=1}^{\infty} \alpha_{k} \theta_{k} z^{k},|z|<\delta\left(f_{\theta}\right)$, then $\left(f_{\theta}(z)\right)^{q}=(1+x)^{q}$, and this implies

$$
d_{\theta \ell}=\left(\begin{array}{l}
q \\
1
\end{array}\right) \alpha_{\ell} \theta_{l}+\left(\begin{array}{l}
q \\
1
\end{array}\right)\left(\alpha_{1} \alpha_{l-1} \theta_{1} \theta_{l-1}+\alpha_{2} \alpha_{l-2} \theta_{2} \theta_{\ell-2}+\ldots\right)+\ldots+\left(\begin{array}{l}
q \\
l
\end{array}\right) \alpha_{1}^{\ell} \theta_{1}^{\ell} .
$$

Hence we have a representation of the form:

$$
d_{\theta \ell}=\sum_{n+\ldots+n} \leq \ell \gamma_{j}\left(\alpha_{1} \theta_{1}\right)^{n_{1 j}} \ldots\left(\alpha_{\ell} \theta_{\ell}\right)^{n_{\ell j}} \text {, }
$$

where $\gamma_{j} \in R$ and depends only on $q$ (not on $\left.\theta\right)$. If $Y_{n, l}(1 \leq n \leq \ell)$ is the coefficient of $z^{l}$ in $\left(\alpha_{1} z-\alpha_{2} z^{2} \alpha_{3} z^{3}-\ldots\right)^{n}$ then $Y_{n, l}$ is a sum of monomials of the form $(-1)^{l-n} \alpha_{1_{1}} \ldots \alpha_{1_{n}}, i_{1}+\ldots+i_{n}=l$. But sgn $\left(\begin{array}{l}q \\ n\end{array}\right)=$ $\left\{\begin{array}{l}(-1)^{n+1}, 0<q<1 \\ (-1)^{n}, q<0\end{array}\right.$ and by (1) we have

$$
d_{\psi \ell}=\sum_{n=1}^{\infty}\left(\begin{array}{c}
q \\
n
\end{array}\right) Y_{n, l} .
$$

Hence using the representation (2) we have

$$
\left|d_{\psi \ell}\right|=\sum_{1 j} \sum_{\ldots+n_{\ell j} \leq \ell}\left|\gamma_{j}\right| \alpha_{1}^{n_{1 j}} \ldots \alpha_{\ell}^{n_{\ell j}}
$$

and the conclusion therefore follows.

口

PROOF OF THEORMM A. We can assume $m=0, a_{0}>0, a_{1} \geq 0$. Let

$$
(f(z))^{p / 2}=\sum_{k=0}^{\infty} d_{k} z^{k},|z|<\delta(f) \text { and }\left(f_{1}(z)\right)^{p / 2}=\sum_{k=0}^{\infty} d_{k}^{\prime} z^{k},|z|<\delta_{1}(f) .
$$

By the representation in Proposition 1 we have

$$
\begin{aligned}
& \int_{0}^{2 \pi}\left|f\left(r e^{i \phi}\right)\right|^{p} d \phi=2 \pi \sum_{k=0}^{\infty}\left|d_{k}\right|^{2} r^{2 k}, 0 \leq r<\delta(f) \\
& \int_{0}^{2 \pi}\left|f_{1}\left(r e^{i \phi}\right)\right|^{p} d \phi=2 \pi \sum_{k=0}^{\infty}\left|d_{k}^{\prime}\right|^{2} r^{2 k}, \quad 0 \leq r<\delta\left(f_{1}\right) .
\end{aligned}
$$

By Lemma 2 with $q=\frac{p}{2}<1$ we have $\left|d_{k}\right| \leq\left|d_{k}^{\prime}\right|, k=0,1,2, \ldots$, and we obtain the desired inequality.

For the final part of the theorem we consider the following example:

Fix $0<\alpha<1$ and define $g_{\theta}(z)=\alpha^{2}+2 \alpha z-e^{1 \theta} z^{2}, g_{1}(z)=\alpha^{2}+2 \alpha z-z^{2}$. We have $\delta\left(g_{1}\right)=(\sqrt{2}-1) \alpha$. Denote

$$
G_{1}(r)=\int_{0}^{2 \pi} \frac{d \phi}{\left|g_{1}\left(r e^{1 \phi}\right)\right|}, \quad G_{\theta}(r)=\int_{0}^{2 \pi} \frac{d \phi}{\left|g_{\theta}\left(r e^{1 \phi}\right)\right|} .
$$

Then $G_{1}\left(\delta\left(g_{1}\right)\right)=G_{\theta}\left(\delta\left(g_{\theta}\right)\right)=+\infty$. Since $\delta\left(g_{1}\right)<\delta\left(g_{\theta}\right)$ for all $0 \supsetneqq \theta \npreceq 2 \pi$ and $\lim _{\theta \rightarrow 0^{+}} \delta\left(g_{\theta}\right)=\delta\left(g_{1}\right)$, we can do the following: 
For every $r_{0}>\delta\left(g_{1}\right)$ we can choose a $\theta_{0}>0$ such that $\delta\left(g_{1}\right)<\delta\left(g_{\theta_{0}}\right) \leq r_{0}$. So $G_{1}\left(r_{0}\right)<\infty$ while $G_{\theta_{0}}\left(r_{0}\right)$ could be made as large as we want and in particular we can choose $\theta_{0}$ so that $G_{1}\left(r_{0}\right)<G_{\theta_{0}}\left(r_{0}\right)$ 口

PROOF OF THEOREM B. Let $\left(f_{\alpha}(z)\right)^{p / 2}=\sum_{k=0}^{\infty} d_{k} z^{k},|z|<\delta\left(f_{\alpha}\right),\left(f_{\beta}(z)\right)^{p / 2}=$ $\sum_{k=0}^{\infty} d_{k}^{\prime} z^{k},|z|<\delta\left(f_{\beta}\right)$. By representation in Proposition 1 it suffices to prove that $\left|d_{k}\right| \leq\left|d_{k}^{\prime}\right|, k=0,1,2, \ldots$. By by formula (2) in the proof of Lemma 2 we have $\left|d_{k}\right|=\sum\left|\gamma_{j}\right| \alpha_{1}{ }^{n_{1 j}} \ldots \alpha_{k}^{n_{k j}},\left|d_{k}^{\prime}\right|=\sum\left|\gamma_{j}\right| \beta_{1}{ }^{n_{1 j}} \ldots \beta_{k}{ }^{n_{k j}}$ and we are done.

The combination of Theorems $A$ and $B$ gives us, for example, the following:

COROLLARY 3. Let $p<2$ and let $\theta$ be an infinite complex vector of modulus 1 components.

(a) For every $f \in P$ and every $0 \leq r<\frac{1}{3}$ we have

$$
\int_{0}^{2 \pi}\left|f_{\theta}\left(r e^{i \phi}\right)\right|^{p} d \phi \leq \int_{0}^{2 \pi}\left|\frac{1-3\left(r e^{1 \phi}\right)}{1-\left(r e^{i \phi}\right)}\right|^{p} d \phi .
$$

Moreover for every $n \geq 0$ there is a $\delta_{n}>0$ such that for all $0 \leq r<\delta_{n}$ we have

$$
\int_{0}^{2 \pi}\left|f_{\theta}^{(n)}\left(r e^{1 \phi}\right)\right|^{p} d \phi \leq \int_{0}^{2 \pi}\left|\ell^{(n)}\left(r e^{1 \phi}\right)-2 \cdot n !\right|^{p} d \phi \quad\left(\ell(z)=\frac{1+z}{1-z}\right) .
$$

(b) For every $f \in S$ and every $0 \leq r<\frac{1}{2}(2-\sqrt{2})$ we have

$$
\int_{0}^{2 \pi}\left|f_{\theta}\left(r e^{1 \phi}\right)\right|^{p} d \phi \leq \int_{0}^{2 \pi}\left|\frac{\left(r e^{1 \phi}\right)}{\left(1-\left(r e^{1 \phi}\right)\right)^{2}}-2\left(r e^{i \phi}\right)\right|^{p} d \phi \text {. }
$$

If $\delta$ is the minimal modulus of the zeros of $1+z-2(1-z)^{3}$ then for every $0 \leq r<\delta$ we have

$$
\int_{0}^{2 \pi}\left|f_{\theta}^{\prime}\left(r e^{1 \phi}\right)\right|^{p} d \phi \leq \int_{0}^{2 \pi}\left|\frac{1+\left(r e^{1 \phi}\right)}{\left(1-\left(r e^{1 \phi}\right)\right)^{3}}-2\right|^{p} d \phi
$$

Finally for every $n \geq 1$ there is a $\delta_{n}>0$ such that for all $0 \leq r<\delta_{n}$ we have

$$
\int_{0}^{2 \pi}\left|f_{\theta}^{(n)}\left(r e^{1 \phi}\right)\right|^{p} d \phi \leq \int_{0}^{2 \pi}\left|k^{(n)}\left(r e^{1 \phi}\right)-2 n \cdot n !\right|^{p} d \phi \quad\left(k(z)=\frac{z}{(1-z)^{2}}\right) .
$$

COROLLARY 4. Let $f(z)=\sum_{k=0}^{\infty} a_{k} z^{k} \in H(U), a_{0} \neq 0$ and $f_{1}(z)=-\left|a_{0}\right|+\sum_{k=0}^{\infty}\left|a_{k}\right| z^{k}$, then for every $p \geq 0$ and for all $0 \leq r<\min$ $\left(\delta\left(f_{1}\right), \delta\left(\frac{2}{f_{1}(0)}-\frac{1}{f_{1}(z)}\right)\right.$ we have

$$
\int_{0}^{2 \pi}\left|f\left(r e^{i \phi}\right)\right|^{p} d \phi \leq \int_{0}^{2 \pi}\left|\frac{f_{1}(0) f_{1}\left(r e^{1 \phi}\right)}{2 f_{1}\left(r e^{i \phi}\right)-f_{1}(0)}\right|^{p} d \phi
$$


PROOF. Since $\frac{1}{f(z)}=\frac{1}{a_{0}}+\sum_{k=1}^{\infty} \alpha_{k} z^{k}, \frac{1}{f_{1}(z)}=-\left(\frac{1}{a_{0} T}+\sum_{k=0}^{\infty}\left|\beta_{k}\right| z^{k}\right)$ and both are analytic in $D\left(0, \delta\left(f_{1}\right)\right)$ and $\left|\alpha_{k}\right| \leq\left|\beta_{k}\right|, \quad k=1,2,3, \ldots$, we obtain

$$
\int_{0}^{2 \pi}\left|f\left(r e^{i \phi}\right)\right|^{p} d \phi \leq \int_{0}^{2 \pi}\left|\frac{1}{f\left(r e^{i \phi}\right)}\right|^{-p} d \phi \leq \int_{0}^{2 \pi}\left|\frac{2}{f_{1}(0)}-\frac{1}{f_{1}\left(r e^{i \phi}\right)}\right|^{-p} d \phi
$$

for every $0 \leq r<\min \left(\delta\left(f_{1}\right), \delta\left(\frac{2}{f_{1}(0)}-\frac{1}{f_{1}(z)}\right)\right.$.

PROOF OF THEOREM C. Let $f(z)=z^{m} h(z)$ where $h \in H(U)$ and $h(0) \neq 0$. As usual let $(h(z))^{p / 2}=\sum_{k=0}^{\infty} d_{k} z^{k},|z|<\delta(f)$. Then, just as in Proposition 1 , we have

$$
\int_{0}^{2 \pi}\left|f\left(r e^{i \phi}\right)\right|^{p} e^{-i n \phi} d \phi=2 \pi\left(\sum_{k=0}^{\infty} d_{k+n} \bar{d}_{k} r^{2 k}\right) r^{m p}, \quad 0 \leq r<\delta(f) .
$$

For $|z|$ small enough we have, as in Lemma 2:

$$
\begin{aligned}
\left(1-\alpha_{1} z-\alpha_{2} z^{2}-\alpha_{3} z^{3}-\ldots\right)^{q} & =1+\left(-q \alpha_{1}\right) z+\left[-q \alpha_{2}+\frac{q(q-1)}{2 !} \alpha_{1}^{2}\right] z^{2} \\
& +\left[-q \alpha_{3}+\frac{q(q-1)}{2 !}\left(2 \alpha_{1} 2 \alpha_{2}\right)-\frac{q(q-1) q(q-2)}{3 !} \alpha_{1}^{3}\right] z^{3} \\
& +\left[-q \alpha_{4}+\frac{q(q-1)}{2 !}\left(2 \alpha_{1} \alpha_{3}+\alpha_{2}^{2}\right)-\frac{q(q-1) q(q-2)}{3 !}\left(3 \alpha_{1}^{2} \alpha_{2}\right)\right. \\
& \left.+\frac{q(q-1) q(q-2)(q-3)}{4 !} \alpha_{1}^{4}\right] z^{4}+\ldots
\end{aligned}
$$

and if $d_{k}$ is the coefficient of $z^{k}$ then $\max \left|d_{k}\right|$ is solved simultaneously for all $k=0,1,2, \ldots$ if we choose $\arg \left(\alpha_{j}\right)=0, j=1,2,3, \ldots$ when $q<1$. When considering $\left.\max _{\theta}\left|\int_{0}^{2 \pi}\right| f_{\theta}\left(\mathrm{re}^{1 \phi}\right)\right|^{\mathrm{p}} \mathrm{e}^{-\mathrm{in} \phi} \mathrm{d \phi} \mid$, we naturally would like to solve simultaneously

$$
\max _{\boldsymbol{\theta}}\left|\mathrm{d}_{\mathbf{k}}\right| \text { and } \arg \left(\mathrm{d}_{\mathbf{k}+\mathbf{n}} \overline{\mathrm{d}}_{\mathbf{k}}\right) \equiv \text { const., } \mathbf{k}=0,1,2, \ldots .
$$

So let us check $\arg \left(d_{k+n} \bar{d}_{k}\right)$ for the choice $\arg \left(\alpha_{j}\right)=0, j=1,2,3, \ldots$ If $0 \leq q<1$ then $\operatorname{sgn}\left(d_{0}\right)=+1$ while $\operatorname{sgn}\left(d_{k}\right)=-1, k=1,2,3, \ldots$. If $q<0$ then $\operatorname{sgn}\left(d_{k}\right)=+1, k=0,1,2, \ldots$. This proves that when $q<0$, the choice $\arg \left(\alpha_{j}\right)=0, j=1,2,3, \ldots$ solves (1) and the conclusion follows.

The proof of Theorem D goes along these lines in analogy to the proof of Theorem B.

\section{REFERENCES}

[1] G.H. Hardy and J.E. Littlewood, "Notes on the theory of series (XIX): a problem concerning majorants of Fourier series", Quart. J. Math. (Oxford) 6 (1935), 304-315.

[2] H.S. Shapiro, "Majorant problems for Fourier coefficients", Quart. J. Math. (Oxford) (2), 26 (1975), 9-18. 


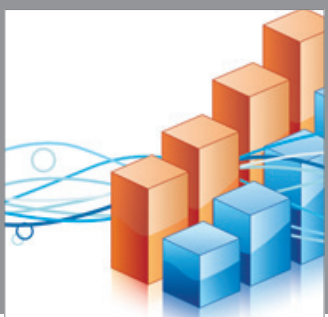

Advances in

Operations Research

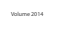

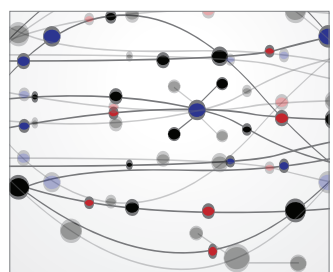

\section{The Scientific} World Journal
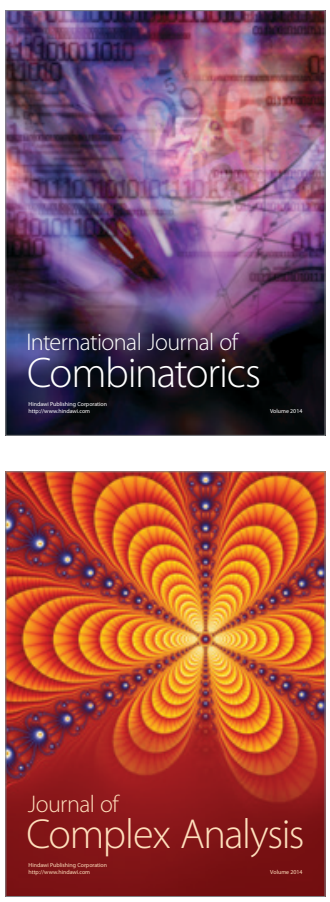

International Journal of

Mathematics and

Mathematical

Sciences
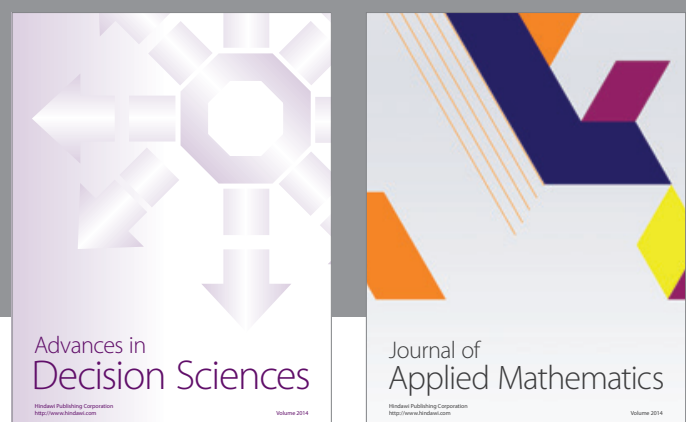

Journal of

Applied Mathematics
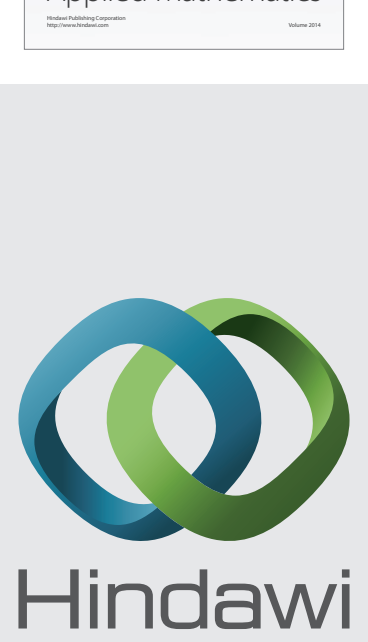

Submit your manuscripts at http://www.hindawi.com
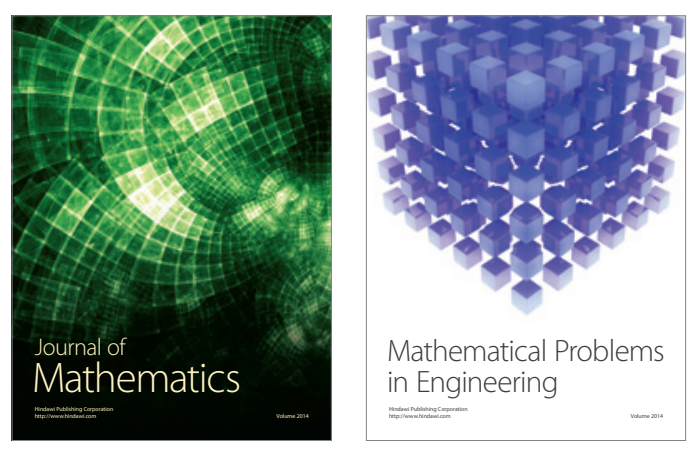

Mathematical Problems in Engineering
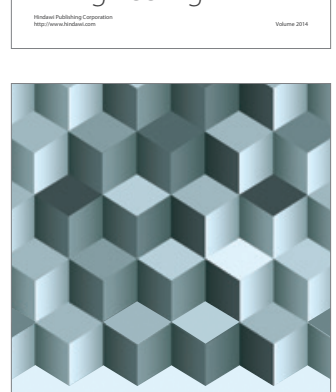

Journal of

Function Spaces
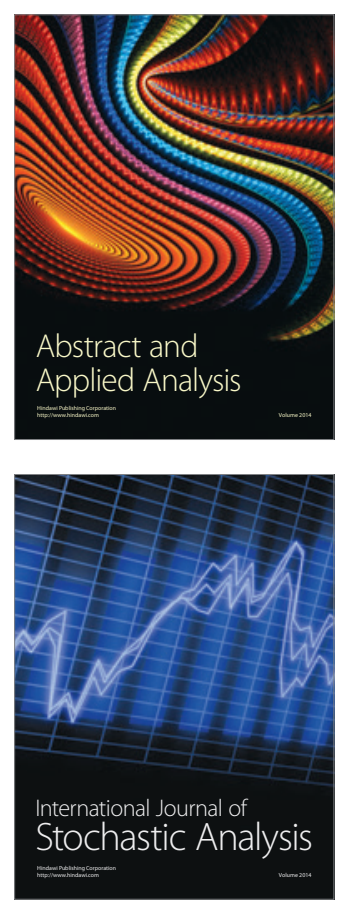

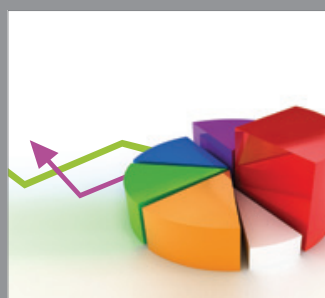

ournal of

Probability and Statistics

Promensencen
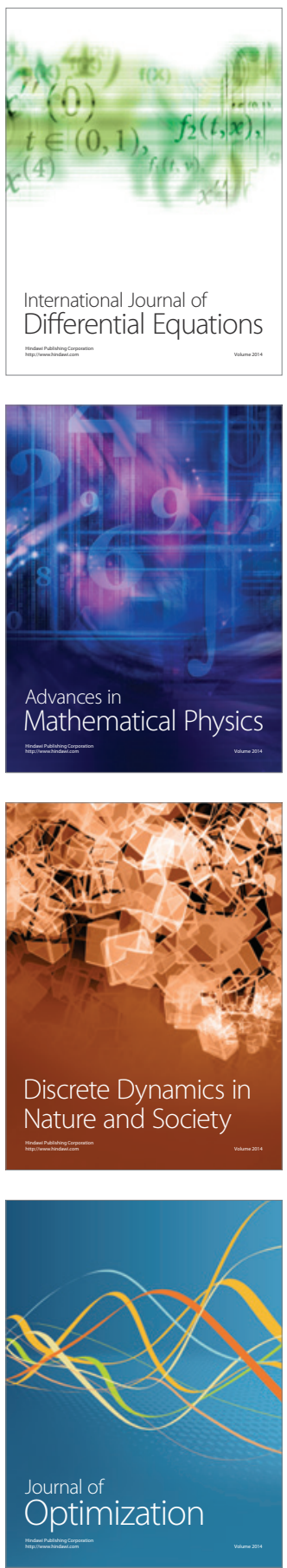